\title{
PRECISION CURRENT REGULATION OF MULTIPOLE MAGNETS USING COMMERCIAL SMPS
}

\author{
Coles Sibley, Oscar Calvo and Tomás Russ \\ Massachusetts Institute of Technology \\ Bates Linear Accelerator Center \\ PO Box 846, Middleton Ma, 01949
}

\section{ABSTRACT}

The South Hall Ring (SHR) under construction at the MIT-Bates Linear Accelerator Center will provide a high duty factor electron beam and internal target capability for nuclear research. For proper operation of the pulse stretcher ring, a large number of highly stable power supplies are required. Most of these supplies will be powering multipole magnets at currents ranging from 10 A. to $155 \mathrm{~A}$. with the output power between 1.2 and 11.5 $\mathrm{KW}$. The high stability specifications of these supplies. better than 25 PPM of the 1.1 GEV current value, including temperature drift and ripple, normally suggests the purchase of custom manufactured supplies. However, due to the costs of custom supplies, we tested the feasibility of upgrading commercial supplies with an external controller to improve the current regulation from a typical value of 200 PPM to better than 25 PPM.

Several upgrade configurations were tested. The results of those tests are discussed as well as some data on the final design.

\section{INTRODUCTION}

The South Hall Ring project under construction at Bates Linear Accelerator Center will require over 80 power supplies for the multipole elements. Some of the quadrupoles and sextupoles will be run in series to cut down on the number of supplies. Their stability, including ripple, noise and drift, must be better than 25 PPM over a temperature span of $10^{\circ} \mathrm{C}$. Usually this type of power supply is custom made and costs from 2 to 3 times more than an "off the shelf" supply of similar output power. Due to the cost considerations we opted to test several supplies, both linear and switching, with an external upgrade to improve the stability specifications to better than 25 PPM. After testing several configurations, we settled on a design which uses two control lowps, a voltage loop which is inside the supply to reduce the ripple to acceptable levels and an external control loop which regulates the current loop and keeps the drift within tolerances. This approach has been tested and regulates the supplies within the tight stability specifications required for the successful operation of the South Hall Ring.

\section{DESIGN APPROACH}

It is easily shown that the components which affect the stability drift performance are the shunt, analog reference, and the error amplifier. We opted to place these components on an external controller board with low drift devices. Once this is done, the greatest effect on the ripple and noise specifications are given by the gain of the feedback loop and good shielding and grounding practices. In the first configuration that was tested, our error amplifier output was "ORed" with the supply's voltage and current regulation loop. The shunt was placed directly in series with the internal shunt, within the output capacitor circuit, thus seeing a "fast" current being shorted through the capacitor and a "slow" current from the load. The loop transfer curves are shown in figure 1.

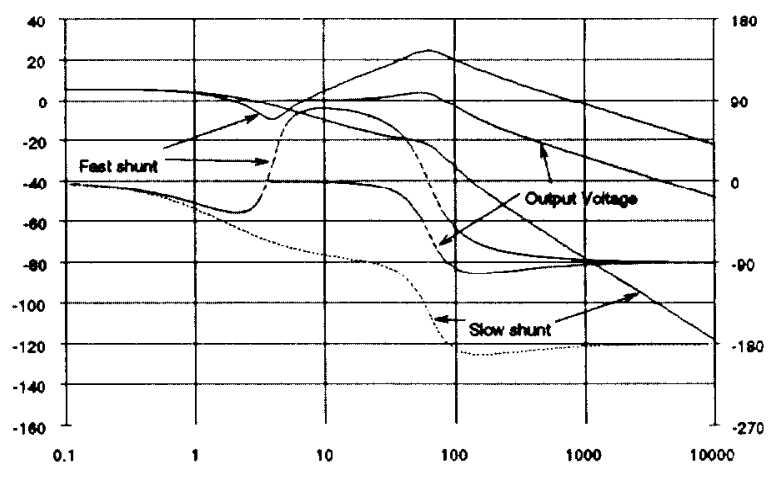

Figure 1. Spice model of a Bode plot for the shunt currents and output voltage

In this case an integrator with a simple zero-pole pair compensates for the load and filter. The required rejection at the line frequencies was easy to obtain. The problem with this configuration is that the leakage current through the capacitor changes with temperature, so we would see drifts in the output current due to room temperature changes. We then moved the shunt outside the capacitor so it would be regulating only on the load current. This solves the drift problem, but the resulting 
current transform has an additional pole, thus increasing the complexity of the feedback loop. In this configuration it is hard to get enough rejection of the line ripple. The final setup was to use the original voltage loop, with minor adjustments in the feedback to reject the line noise. The output of our regulation loop is then used as the reference into the voltage loop. This gives us a fast inner loop which compensates for line ripple and noise, and a slow outer loop for control of the current stability.

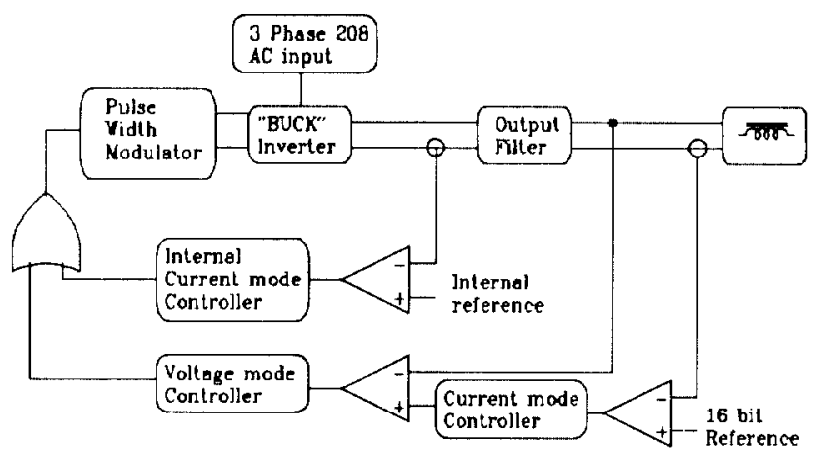

Figure 2. Block diagram of power supply control loops.

\section{SYSTEM OVERVIEW}

The power supply consists of three separate systems: the commercial power supply, the analog board and the digital board. The power supplies are manufactured by Electronic Measurements, Inc. in Neptune NJ. They are powered in the constant voltage mode, using the error signal from the analog board as the voltage reference. The current control mode acts as a local current limit.

The Bates analog board [1] contains the DAC, three $A D C$ 's, the shunt amplifier, the error amplifier, and the interface to the digital board. The analog board is galvanically isolated from the digital board. The shunt has a flow switch used as a power supply interlock in the digital board. Control signals, the DAC setting and the three ADC signals are run serially through optocouplers for isolation. Power coming from the digital board goes through dc to dc converters for isolation.

The digital board contains a Bitbus interface for remote control, the serial interface between the analog board and the digital board, the status from the power supply, the control logic for the power supply DC on/off, power supply interlock resetting, and the magnet interlocks. There is a microcontroller (8044) which takes care of the communications and timing. Ramping and cycling procedures are handled by the microcontroller.

\section{POWER SUPPLY CONFIGURATION}

The EMS "DCS" series power supplies are switching mode power converters with output power ranging from 600 watts to over 10 Kilowatts. The power supplies overall regulation is about $0.1 \%$.

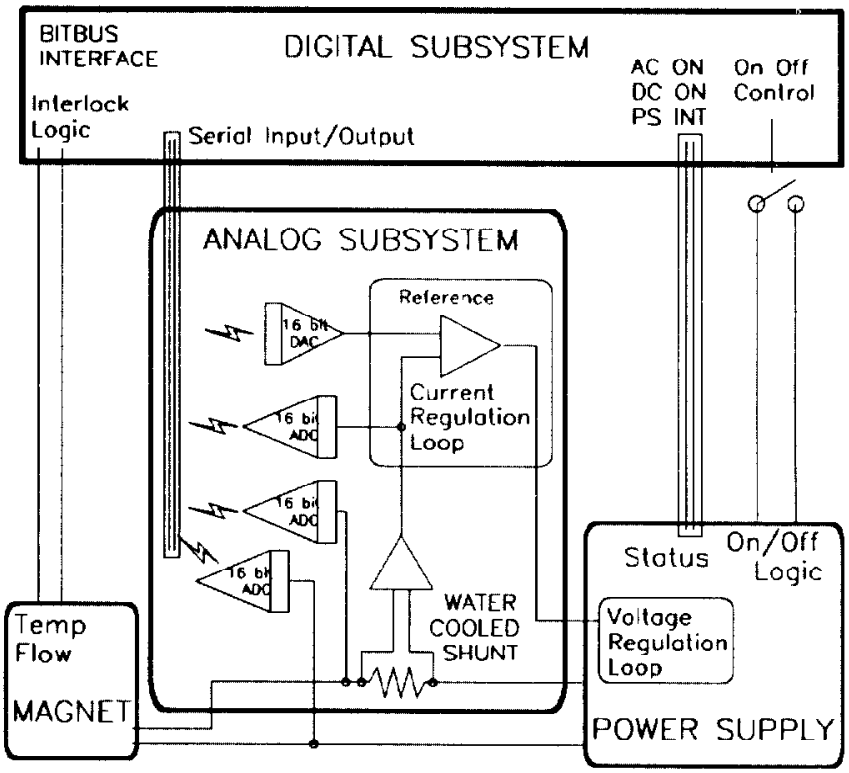

Figure 3. Block diagram of the power supply system.

The basic hardware configuration is the same for both the voltage and current modes of operation. A reference signal is compared with a sample of either the voltage or current, and the difference is amplified through an error amplifier. Both error amplifiers have an integrator and a zcro-polc configuration. The feedback is designed to be stable when powering a wide range of loads. The output of the voltage error amplifier is then ORed with the output of the current error amplifier and a shutdown circuit such that any one can shut down the PWM but only one will regulate at a time, depending on the load resistance. The PWM drives a full bridge configured as a buck converter. For the 5 and $10 \mathrm{KW}$ supplies, two or four bridges are run in parallel to double or quadruple the current capacity.

\section{CONTROLLER CONFIGURATION}

In order to meet the stability specifications, an external shunt and a stable reference are added to the system. The voltage reference is a Thalor precision $5 \mathrm{v}$ reference with a temperature coefficient of better than $.6 \mathrm{PPM} /{ }^{\circ} \mathrm{C}$ and line regulation specifications of better than $2 \mathrm{PPM}$. / V. The DAC is 16 bits and has a long term drift of less than $1 \mathrm{PPM} /{ }^{\circ} \mathrm{C}$ and offset of better than $.5 \mathrm{PPM} /{ }^{\circ} \mathrm{C}$. The shunts are Zeranin shunts with a tolerance of .1 percent. The temperature coefficient is 3 PPM over a temperature range of 0 to $60^{\circ} \mathrm{C}$. They are water cooled with an input water temperature of $30^{\circ} \pm 1^{\circ} \mathrm{C}$ and can dissipate up to 200 watts. The shunt signal goes through a common mode filter to kill any switching frequency noise with a cutoff frequency of about $600 \mathrm{~Hz}$ and is then amplified by an instrumentation amplifier (an LT1101) with a fixed gain of 10, then amplified again (Av 1.2) to have an output of 10 volts at the supplies full output current. The second amplifier has the necessary offset and gain adjustment for calibration purposes. This signal is routed to the front 
panel for monitoring, and also to a voltage divider whose output goes to the error amplifier and to a 16 bit ADC. This circuit is shown in Fig. 4. The output of the error amplifier goes into the voltage channel reference input of the power supply. The shield of this cable is where the common of the supply is tied to the common of our analog circuit. All shields coming in to the analog board are tied at the same place on the ground plane of the analog board to eliminate any ground loops.

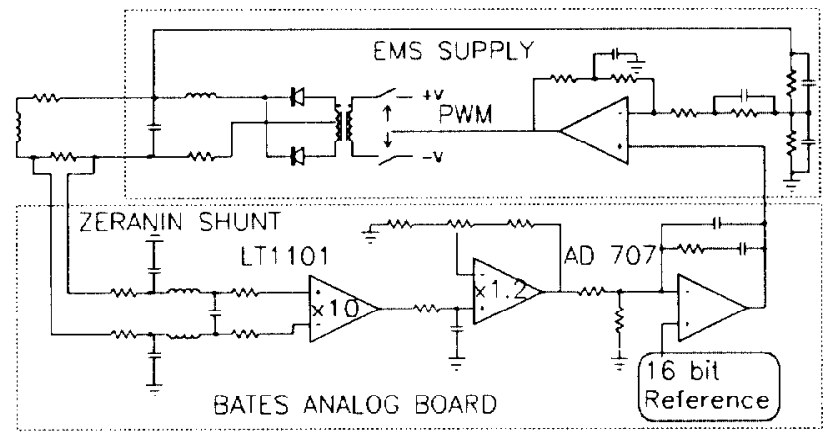

Figure 4. Schematic of voltage and current control loops

\section{VOLTAGE LOOP}

The stability specification of 25 PPM includes all ripple and noise. The worst case for ripple is at $60 \mathrm{~Hz}$ with a single quadrupole as the load. After calculating the maximum ripple from each supply and comparing that with the maximum current ripple allowable, we find that each supply's voltage feedback loop has to be modified slightly. Table 1 shows the minimum amount of gain boost required for each supply to bring the ripple and noise specifications into tolerance. From this table, we see that if we boost the voltage loop gain by 10 to $20 \mathrm{~dB}$, we will more than cover all the cases for the ripple specifications. Fig. 5 shows the measurcd loop response, with a quadrupole as the load, for the EMS loop and the modificd voltage loop. The supply used in this example was an EMS 60-80. Figure 4 shows the modified voltage feedback circuit. In this case, the addition of an integrator in the voltage feedback loop would have been beneficial. This can be implemented with just a change of values in the original EMS design.

\begin{tabular}{|l|l|l|l|l|l|l|l|l|l|}
\hline $\begin{array}{l}\text { Amp } \\
s\end{array}$ & 155 & 110 & 80 & 60 & 60 & 52 & 40 & 40 & 10 \\
\hline Volts & 70 & 95 & 70 & 80 & 40 & 48 & 120 & 30 & 125 \\
\hline
\end{tabular}

\begin{tabular}{|l|l|l|l|l|l|l|l|l|l|}
\hline DB's & 1.6 & 2.7 & 4.2 & 3.2 & 7.7 & 7.7 & .74 & 12.4 & 3.7 \\
\hline
\end{tabular}

\section{Table 1}

With the voltage loop closed at a bandwidth of between 5 and $10 \mathrm{Khz}$, the loop has enough rejection at the line frequency for the stability specifications. The current loop now can be closed at a low enough frequency so the extra pole introduced by the load is above the bandwidth of the circuit and does not affect the current loop stability. Figure 6 shows the current loop response with the voltage loop closed. From this chart it is clear that we will not have any problems with stability as long as we close the loop under $100 \mathrm{~Hz}$.

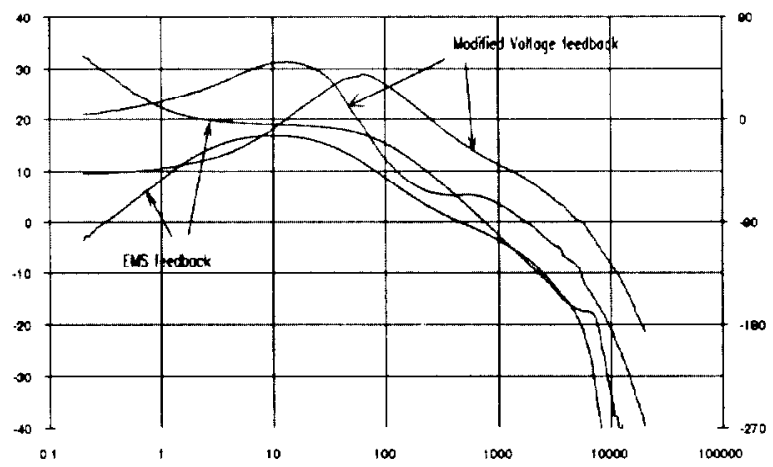

Figure 5. Bode plot comparing the modifies voltage feedback loop with the original feedback loop.

At approximately $360 \mathrm{~Hz}$ there is a resonant circuit due to a mismatch in the input common mode filter. Below this frequency the gain drops rapidly due to the shunt filtering.

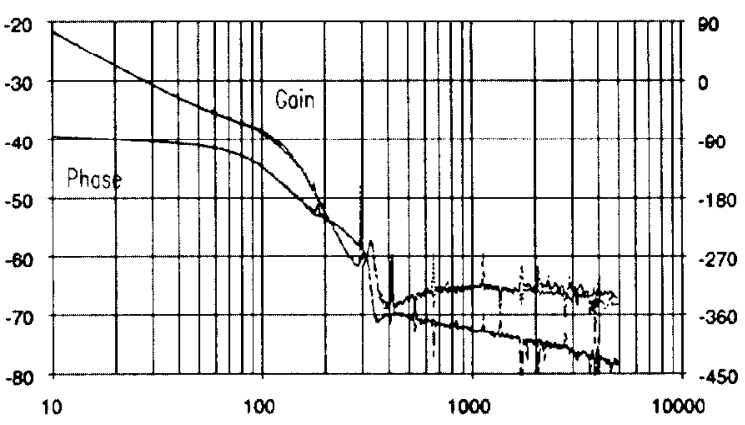

Figure 6. Current loop with voltage loop closed.

\section{CONCLUSION}

The design discussed has been shown to comply with the high stability specifications for the multipole power supplies in the South Hall Ring project. We have ordered 81 power supplies for the quadrupole and sextupole magnets which will utilize this upgrade. The supplies will be upgraded and tested by EMS with deliveries expected in August 1991.

\section{References}

1. T. Russ, C. Sibley, "A distributed Power Supply Controller using the Bitbus Interface", Nuclear Instruments and Methods in Physics Research, A293, 1990

2. O. Calvo, C. Sibley, T. Russ, "High Stability Switching Mode Power Converters for Magnetic Loads.Proceedings of the 1990 Linear Accelerator Conference . p493

3. Instruction Manual for EMS Power Supply EMS 80-60, Electronic Measurements, Neptune, NJ 